\title{
Harmonie in Blau und Gelb - der Majorelle-Garten in Marrakesch
}

\author{
Hilke Steinecke
}

\begin{abstract}
The Jardin Majorelle outside the city walls of Marrakesh, Morocco, are the gardens of the late artist-painter JaCQUEs MajoRELLE (1886-1962) - a passionate amateur botanist. The gardens having deteriorated after MAJORELLE's death, were eventually revived by the fashion-maker Yves ST. Laurent. The dominating color is „Majorelle blue“. Exotic tropical plants from all around the world are cultivated in the impeccably maintained gardens - the compound also including a museum. Here, we report on the garden's history, its structure and design, and on several of the particularly prominent plants grown in these splendid gardens.
\end{abstract}

\section{Zusammenfassung}

Der Majorelle-Garten ist ein Künstlergarten vor der Stadtmauer von Marrakesch. Der Modeschöpfer Yves St. Laurent rettete den vom Maler Majorelle gegründeten und nach dessen Tod verwilderten Garten vor dem Verfall. Im heute sehr gut gepflegten Garten gedeihen tropische Pflanzen aus der ganzen Welt. Geschichte des Gartens, seine Gliederung und einzelne markante Arten werden vorgestellt.

\section{Ein Garten des gärtnernden Malers}

In Marrakesch, der Hauptstadt Marokkos, wird der Besucher schnell vom quirlig-bunten Treiben vereinnahmt. Moscheen, reich verzierte $\mathrm{Pa}$ läste, Basare, fliegende Händler, süß duftende Orangenbäume und kleine maurische Gärten in Innenhöfen erzeugen eine Stimmung wie in der Märchenwelt aus 1001 Nacht. Bedeutende echte islamische Gärten gibt es in Marokko kaum noch, dafür aber ein paar "Garten-Relikte“ aus der französischen Kolonialzeit (1911-1956).
Solche Gärten sind der ideale Ort, sich von dem pulsierenden Leben der Stadt zu erholen.

Ein kleiner, aber sehr feiner und weit über die Landesgrenzen hinaus bekannter Garten ist der Majorelle-Garten in Marrakesch. Er ist vom historischen Stadtzentrum leicht zu Fuß oder in einer Pferdekutsche erreichbar. Der Garten ist

Abb. 1: Der Brunnen ist ein wichtiges gestalterisches Element im Garten.

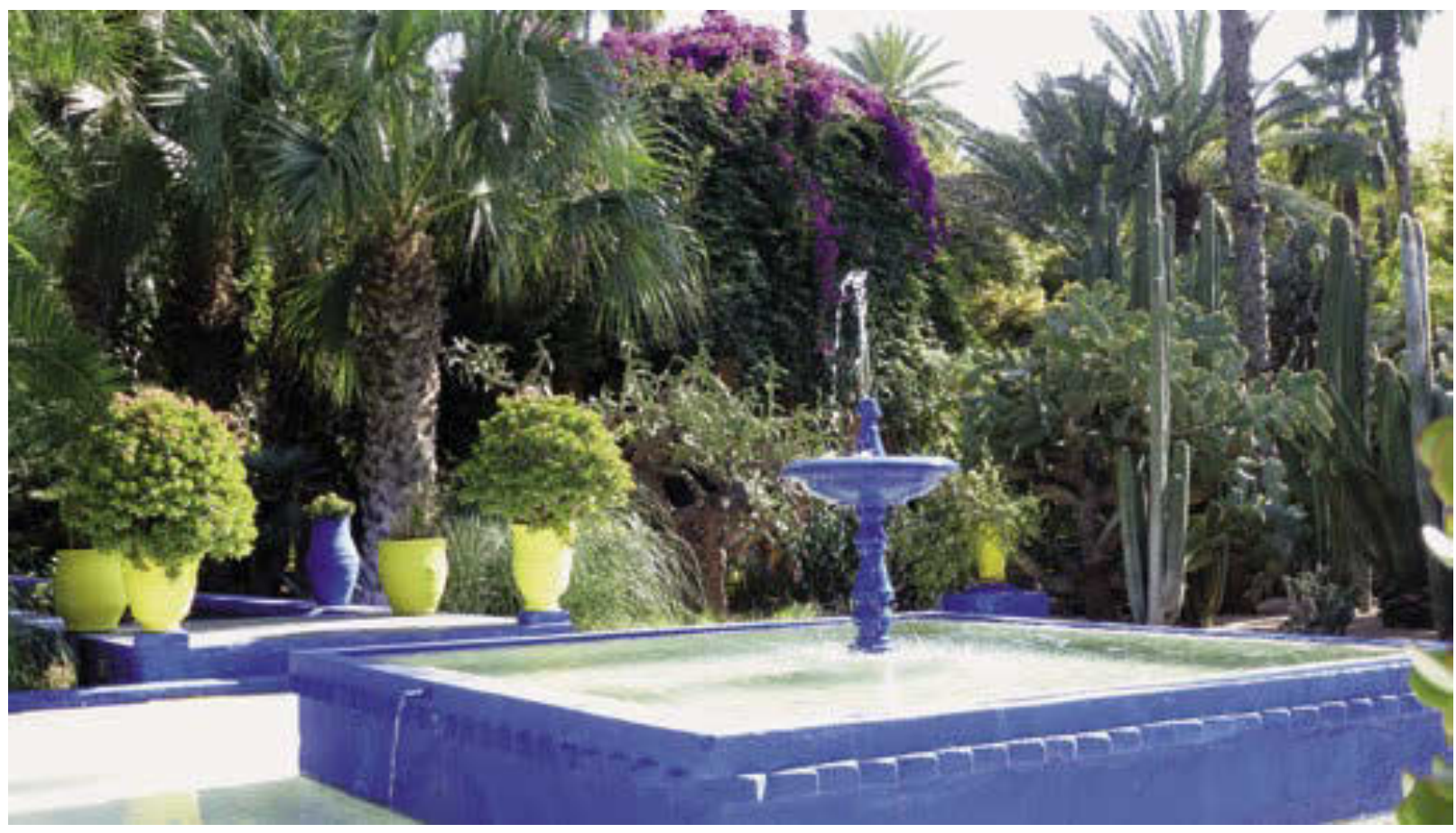




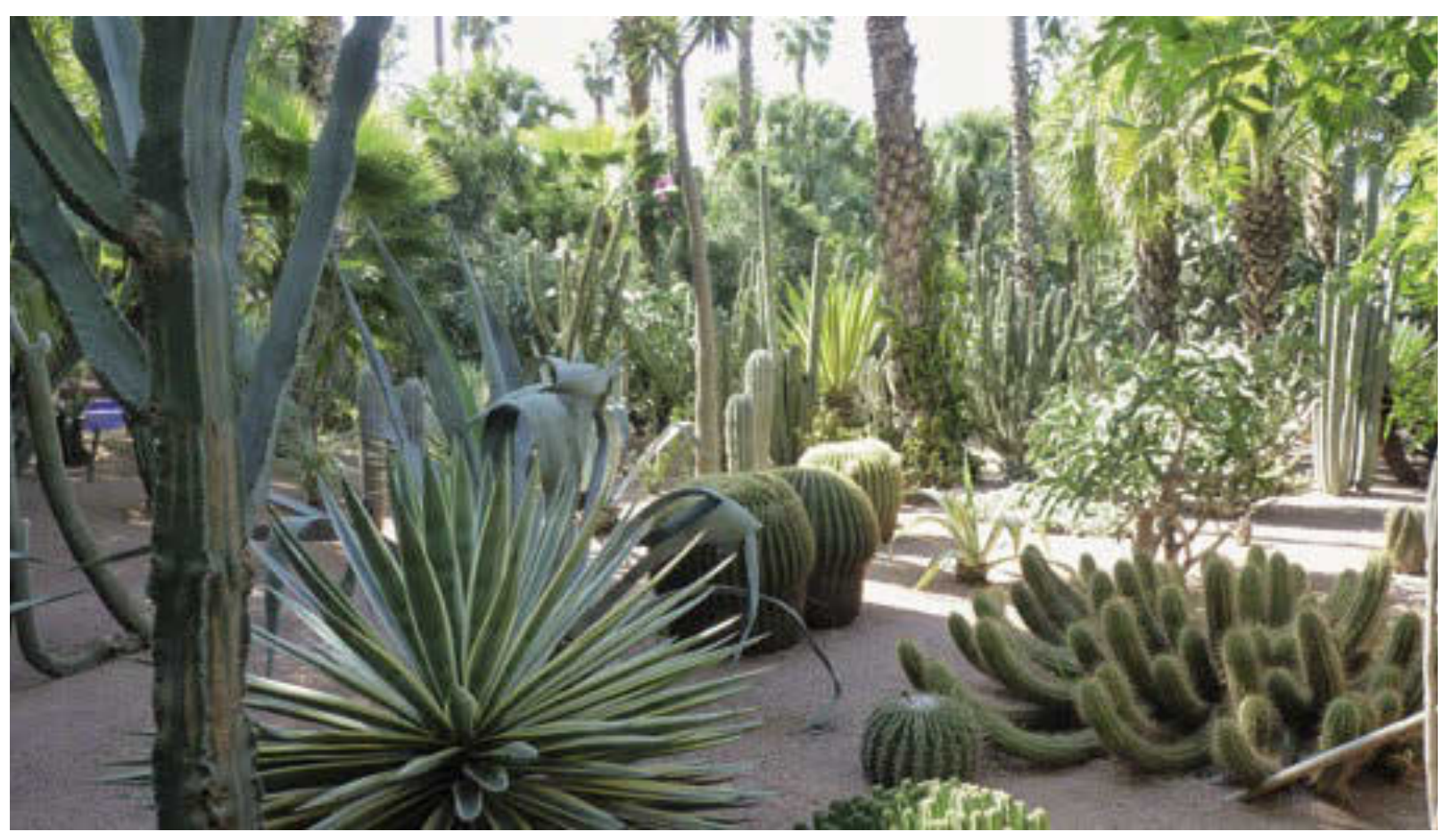

ein Kunstwerk für sich. Erschaffen wurde er von dem französischen Maler Jaques Majorelle (1886-1962), Sohn des Art-Noveau-Künstlers und Möbeldesigners Louis MaJORELLE. JAQUES MajOrelle, sowohl durch den verspielten Jugendstil als auch durch das funktionale Bauhaus beeinflusst, kam 1919 nach Marrakesch. In der Nähe der Stadtmauer konnte er ein etwa 4 ha großes Grundstück erwerben und dort sein eigenes Paradies erschaffen. Der Garten sollte ein dekoratives Gesamtkunstwerk werden, in dem Architektur, Malerei, Kunsthandwerk, Pflanzen und Lebensstil perfekt miteinander harmonieren. Als weit Gereister interessierte sich Majorelle für Kulturen und Pflanzen der Welt, später besonders aus Afrika. Er kultivierte in seinem Garten diverse attraktive Exoten. Inspirieren ließ sich der gärtnernde Künstler durch die Kontraste zwischen Großstadt und den in der Ferne sichtbaren schneebedeckten Gipfeln des Atlasgebirges (Betten 2011). Auch die in der Stadt überall gegenwärtigen Dattelpalmen sowie der ehemals riesige, seit über 1000 Jahren existierende und heute stark bedrohte Dattelpalmenhain rund um Marrakesch gaben ihm vermutlich weitere Anregungen. Besonders beeindruckte ihn aber auch das Bergvolk der Berber und ihre karge Lebensweise.

\section{Geschichte des Gartens}

Nach dem 2. Weltkrieg war Marrakesch für wohlhabende Europäer ein beliebter Aufenthaltsort, viele Prominente aus Politik und öffentlichem Leben kehrten im Majorelle-Garten ein, darunter auch Winston Churchill, um sich seinem Hobby des Malens zu widmen. Majorelle verstarb 1962 in Paris. Er hatte kurz vorher Marokko verlassen, um sich von den Folgen eines schweren Verkehrsunfalls zu erholen, was ihm aber nicht gelang. Der Garten verwilderte, bis er 1980 von dem berühmten französischen Modedesigner Yves Saint Laurent und seinem Lebenspartner Pierre Bergé gekauft und aus dem Dornröschenschlaf geweckt wurde. Für die Restaurierung wurde $a b$ Mitte der 1990er-Jahre der marokkanische Botaniker und führende Gartendesigner sowie Ökologie- und Botanikprofessor an der Universität Marrakesch, Abderrazzak BenchaAbA$\mathrm{NE}$, beauftragt. Der Majorelle-Garten wurde im Sinne seines Gründers wiederhergestellt. Bereits 1997 gründete Bergé eine Stiftung, um den Garten auch für die Zukunft zu erhalten. Yves Saint Laurent ließ sich in seiner aktiven Zeit von den vielen Formen und den intensiven Farben des Gartens für seine Modeschöpfungen inspirieren. Nach seinem Tod im Jahr 2008 
wurde seine Asche im Garten verstreut, eine schlichte Gedenksäule erinnert hier an ihn (Milchert 2010). Heute ist der MajorelleGarten ein sehr beliebtes touristisches Ziel. Etwa 650000 Besucher kommen pro Jahr. Es ist empfehlenswert, den Garten morgens oder abends zu besuchen, weil dann die Touristengruppen noch nicht da oder schon wieder weg sind. Etwa 20 Gärtner hegen und pflegen den Garten, auch eine automatische Bewässerungsanlage ist in Betrieb.

\section{Farbenfroher Garten der Kontraste}

Gleich hinter dem Eingang taucht der Besucher in die grüne Gartenwelt mit ihren blauen und gelben Akzenten ein. Ein kleiner türkis und blau gekachelter Brunnen sorgt für Erfrischung. Mäuerchen, Beeteinfassungen, Bodenbelege der schmalen geschlängelten Wege und Pflanzkübel sind überwiegend in den Komplementärfarben Blau und Gelb gehalten, dazu ein paar rote Kontraste. Diese farbenfrohen Elemente stellen bewusst einen verspielten Gegenpol zu den gedeckten Beige- bis Ockerrottönen der meisten Gebäude Marrakeschs dar, die traditionell aus dem Lehm der Umgebung errichtet wurden. Dieses ganz besondere Kobaltblau, das Majorelle so gern verwendete und den Garten so einmalig macht, wird in Künstlerkreisen auch als Majorelleblau bezeichnet.

Die starken Kontraste des Gartens sind überall wahrnehmbar. Pflanzen aus allen Kontinenten stehen hier dicht nebeneinander, unabhängig von ihren Standortsbedingungen. So gedeihen z. B. nur wenige Meter neben einem dichten Bambushain Agaven (z. B. Agave victoria-reginae, A. stricta, A. macroacantha), Aloen (z. B. A. ferox) und viele Kakteen. Großblättrige Arten aus den feuchten Tropen wie Bananen, Philodendron und Monstera stehen in Nachbarschaft von sukkulenten Palmlilien (z. B. Yucca

Abb. 2 (Seite 86): Sukkulentengarten mit dicken Schwiegermuttersitz-Kakteen.

Abb. 3 (oben): Das blaue Haus des Künstlers.

Abb. 4 (unten): Pflanzen, Kübel und Haus sind farblich aufeinander abgestimmt, hier eine Aloe vera.
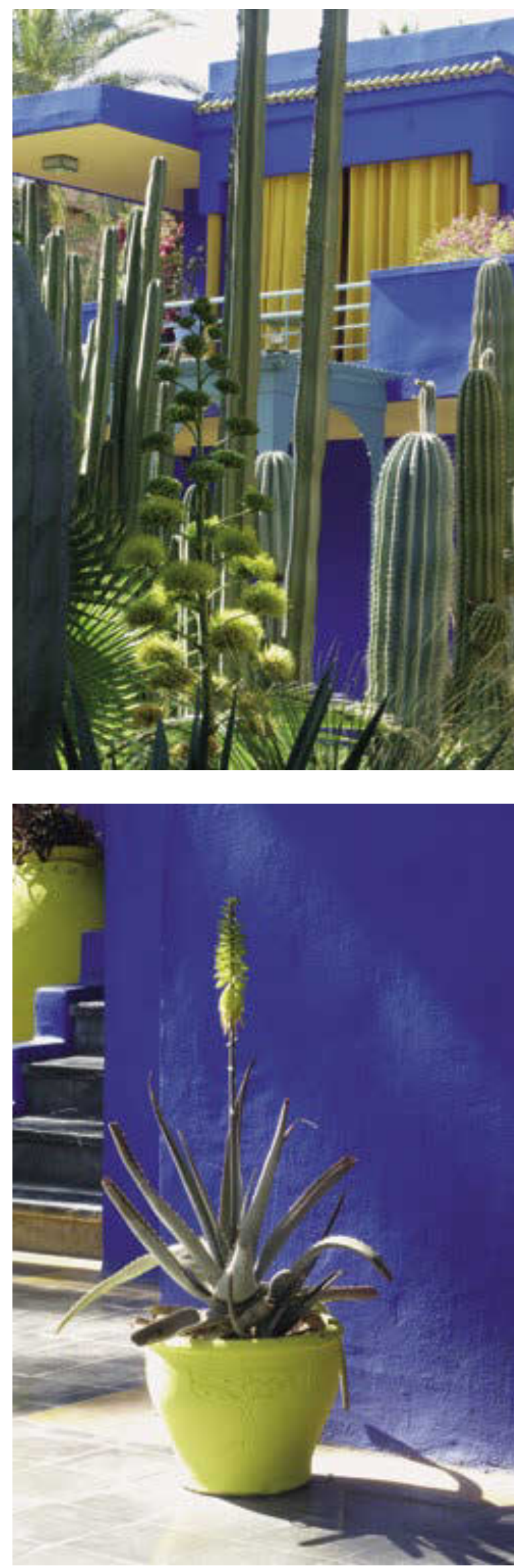

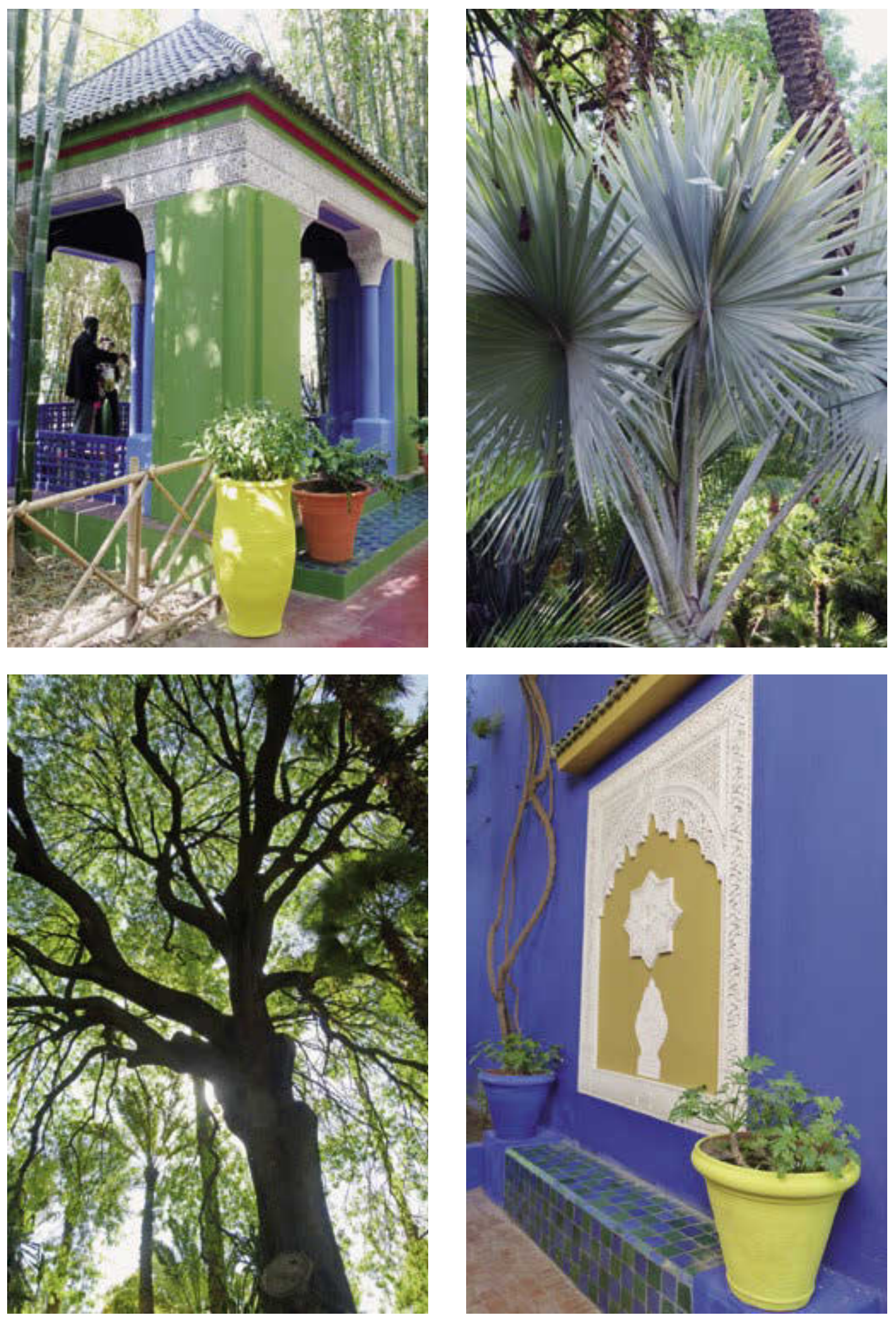
rigida) oder Palmfarnen. Insgesamt soll der Garten etwa 300 verschiedene Pflanzenarten beherbergen. Auf viele von ihnen, aber längst nicht alle, verweisen Infoschilder mit Umrissskizzen und den dazugehörigen wissenschaftlichen Namen.

\section{Markante Pflanzen und Gartenbereiche}

Das markanteste Beet ist sicherlich das zentrale Sukkulentenbeet. Auf einem geschotterten Untergrund gedeihen hier mitunter schon recht große Individuen von Schwiegermuttersitzkakteen (Echinocatus grusonii). Ihre gelben kräftigen Dornen ergänzen das Spiel mit der Farbe Gelb. Weitere markante Kakteen sind hier Echinocereus morricallii, Cylindropuntia tunicata, Oreocereus fossulatus, Pilosocereus azureus und Cephalocereus sessilis. Auch die am höchsten werdenden Kakteenarten Pachycereus pringlei und Carnegiea gigantea sind hier zu finden. Blüten- und Blattfarben harmonieren mit dem blau-gelben Farbkonzept des Gartens, ob es die gelben Blütenstände der Europäischen Zwergpalme, die gelb-grün panaschierten Blätter diverser Agaven oder die blauen Blüten von Petunien und Staticen sind.

Vom Sukkulentengarten fällt der Blick, wie von fast allen Standorten im Garten, auf das 1931 gebaute und Villa Oasis genannte ehemalige Wohnhaus und Atelier Majorelles. Auch dieses Gebäude ist in Blau gehalten mit gelben Fenstereinfassungen, Türen und Vorhängen. Perfekt dazu passen auf der Terrasse platzierte gelbe Blumenkübel mit gelb blühender Aloe vera. Die im Gegenlicht leuchtend rot wirkenden Dornen eines dicken, orangerot blühenden Ferrocactus cf. stainesii greifen die Farbe der we-

Abb. 5 (oben links): Bunter Pavillon am Bambushain.

Abb. 6 (oben rechts): Bismarckia mit ihren großen blaugrauen Fächerblättern.

Abb. 7 (unten links): Dieser Pistazien-Baum gehört vermutlich zu den ältesten Pflanzen im Garten.

Abb. 8 (unten rechts): An die orientalische Architektur angelehnte Ornamente.

Abb. 9 (oben): Große Bougainvilleen mit verschiedenen Blütenfarben kontrastieren mit dem Blau des Hauses.

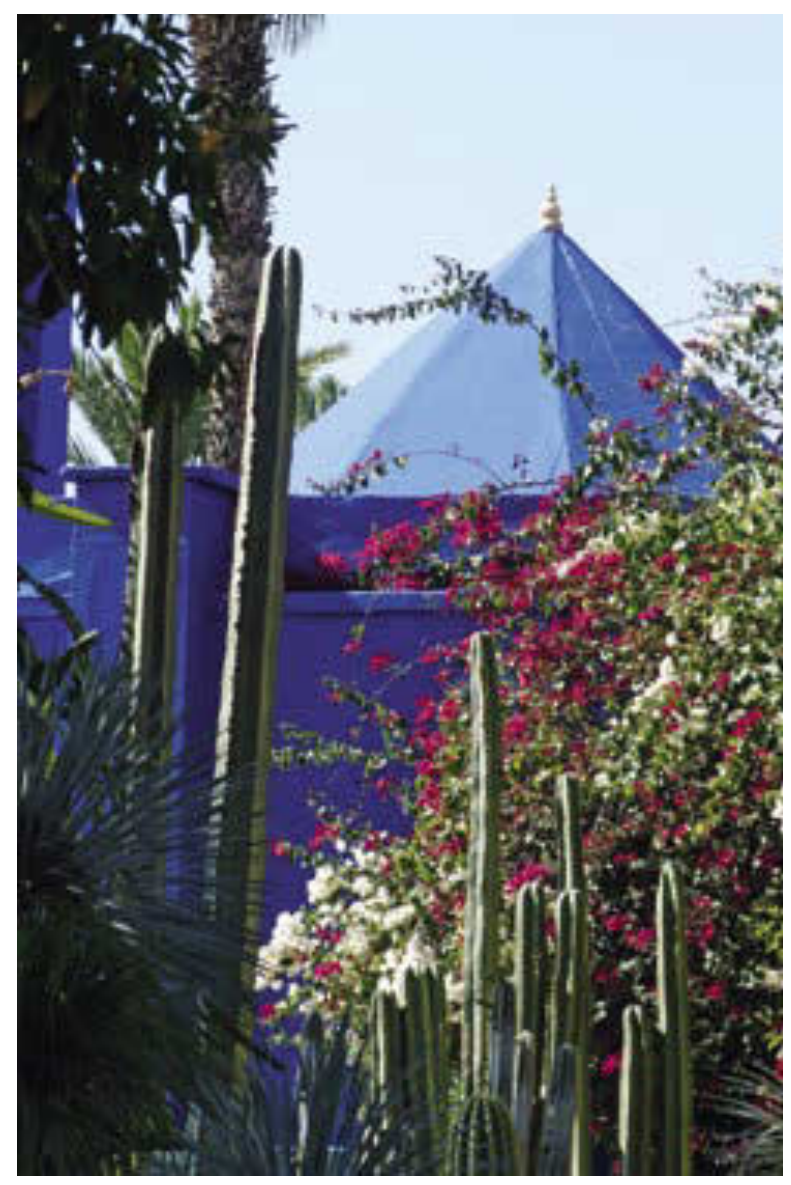

nigen roten Pflanzgefäße auf. Intensiv pink blühende Bougainvilleen am Haus lassen den Farbeindruck noch intensiver erscheinen. Die blaue Villa beherbergt heute ein kleines Museum für Islamische Kunst. Außen ist es mit dekorativen Elementen, die der islamischen Architektur entnommen sind, verziert.

Aus der maurischen Gartenkultur entlehnt ist das längliche, rechteckige Wasserbecken längs des Hauses, das von blauen Säulen flankiert wird. Es wird von einer üppig berankten Pergola und großblättrigen tropischen Aronstabgewächsen umgeben. Blickfang im Garten ist eine blaue Brunnenschale, aus der eine kleine Fontäne spritzt. Das Wasser tröpfelt in ein kleines quadratisches, blau eingefasstes Becken, das sich in der Mitte eines größeren Wasserbeckens befindet. In einem rechteckigen Teich gedeihen Seerosen, allerdings so locker, dass sich noch interessante Spiegeleffekte auf der Wasseroberfläche ergeben. Somit verdoppelt sich hier auch der zumindest im Sommer fast täglich blaue Himmel. Aus der Froschperspektive betrachtet 


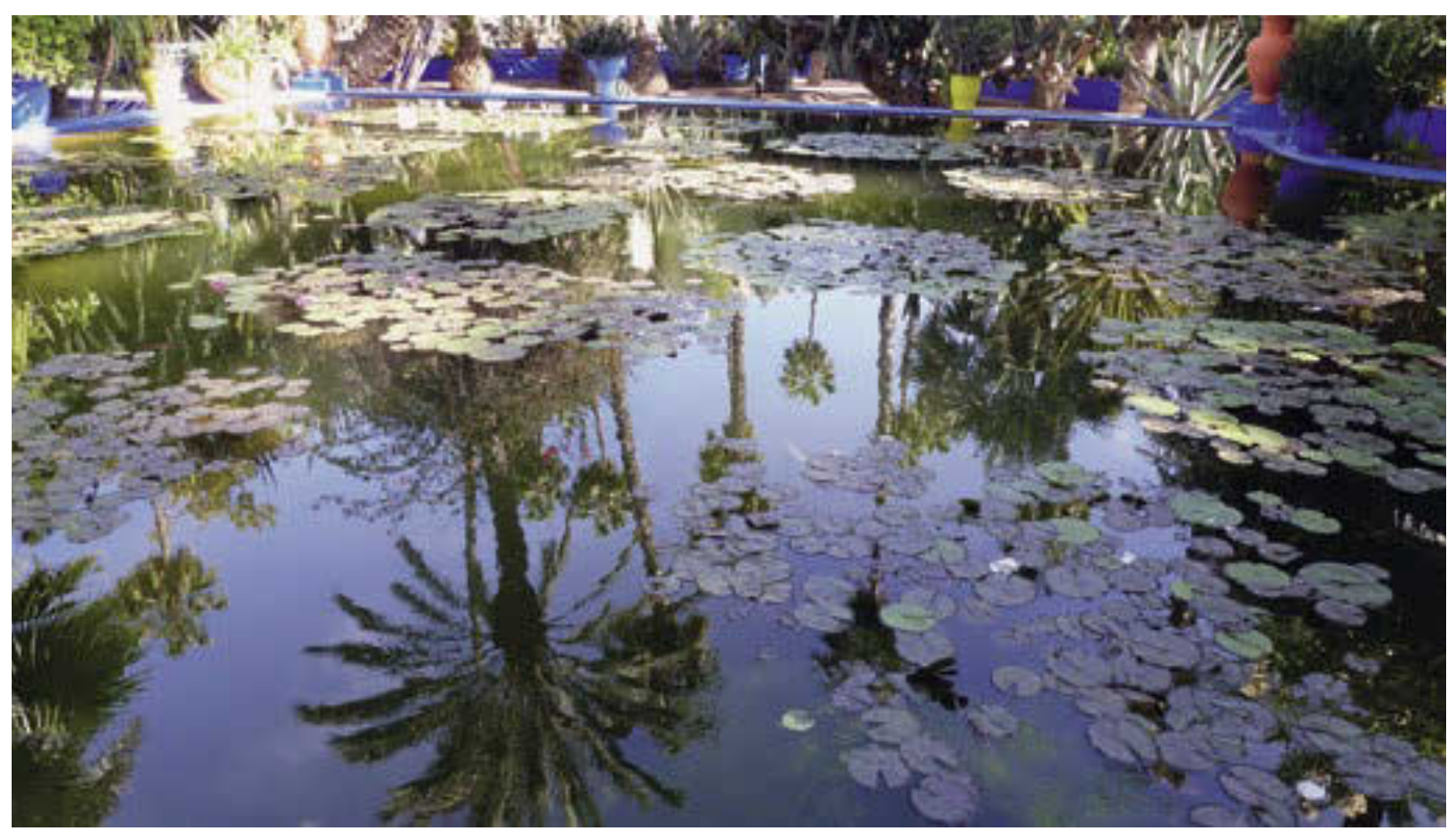

spiegeln sich im Wasser ebenso die schlanken Stämme der hier besonders hohen Dattelpalmen (Phoenix dactylifera). Neben den in Marrakesch allgegenwärtigen Dattelpalmen bereichern Chinesische Hanfpalme (Trachycarpus fortunei), Zwergpalme (Chamaerops humilis), Geleepalme (Butia capitata) und vor allem die attraktive Bismarckpalme (Bismarckia nobilis) den Garten. Letztere stammt aus Madagaskar und fällt durch ihre den Stamm wie Flechtwerk umgebenden Blattscheiden sowie ihre großen blaugrauen Fächerblätter auf. Die Bismarckpalme braucht viel Platz und fällt im Garten durch ihre markanten großen Blätter auf.

\section{Alt und ehrwürdig}

Ein Reiz des Gartens sind seine einzelnen besonders stattlichen Pflanzenindividuen, die aus der Gründerzeit stammen und dementsprechend mindestens rund 100 Jahre alt sein dürften, darunter ein dicker Elefantenfuß (Beaucarnea recurvata), der von einer blauen Beeteinfassung umgeben ist. Vermutlich die älteste Pflanze im Garten, wahrscheinlich schon vor der Anlage des Gartens an ihrem Standort wachsend, ist ein Atlantischer Pistazienbaum (Pistacia atlantica). Diese in Nordafrika, auf den Kanaren und im östlichen Mittel- meerraum heimische Pistazien-Art wird bis 20 Meter hoch. Mit seinen ausladenden Ästen ist der Baum im Majorelle-Garten ein willkommener Schattenspender.

\section{Literatur}

Betten, A. 2011: DuMont Kunst-Reiseführer Marokko: Antike, Berbertraditionen und Islam - Geschichte, Kunst und Kultur im Maghreb. - Köln.

Milchert, J. 2010: Okzident trifft Orient - Jardin Majorelle in Marrakesch. - Gp 4 (2010): 34-39.

\section{Internetseite}

Hansen, D. 2009: Yves Saint Laurent in Marrakesch. Merian, die Lust am Reisen.

http://www.merian.de/magazin/marrakesch-yves-saintlaurent.html

Abb. 10 (oben): Die schlanken Dattelpalmen spiegeln sich im Wasserbecken.

Abb. 11 (Seite 91 oben links): Der Brunnen ist aus verschiedenen Blickwinkeln zu beobachten.

Abb. 12 (Seite 91 oben rechts): Besonders im Gegenlicht leuchten die Dornen dieses Ferrocactus auffällig rot.

Abb. 13 (Seite 91 unten links): Gedenksäule für Yves St. Laurent

Abb. 14 (Seite 91 unten rechts): Nicht nur der Garten bietet Kontraste, sondern auch die Stadt Marrakesch mit Dattelpalmen im Vordergrund und in der Ferne den schneebedeckten Gipfeln des Hohen Atlas. 

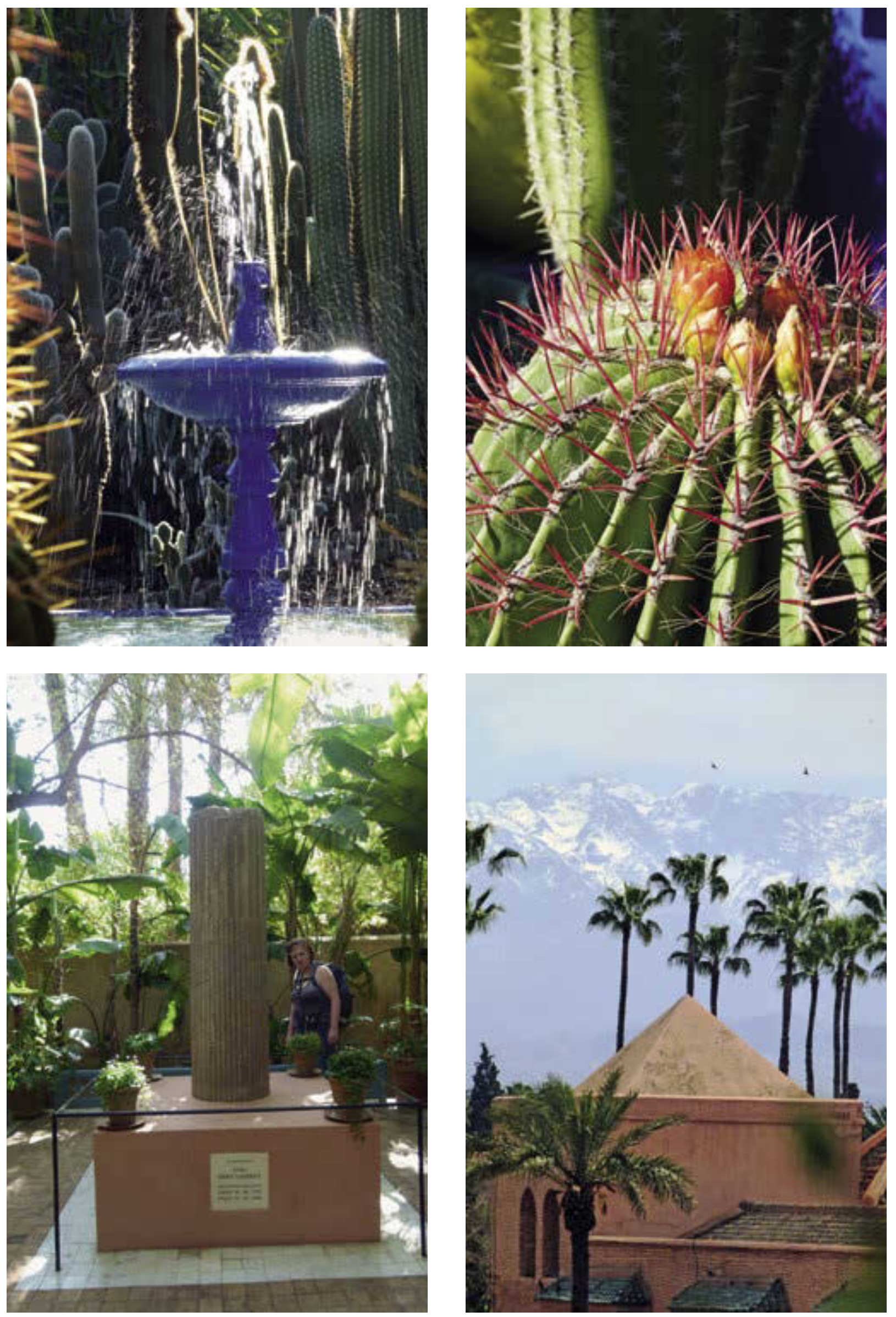